\title{
Electrical Characterization on Signal Transmission of SATA Cable Assembly
}

\author{
Moonjung Kim \\ Division of Electrical Electronic Control Engineering, Kongju National University, \\ Republic of Korea \\ mjkim@kongju.ac.kr
}

\begin{abstract}
Signal transmission analysis is performed for a serial advanced technology attachment (SATA) cable assembly using a three-dimensional (3D) electromagnetic field simulation. In this work, SATA cable assembly consists of the connector, PCB and cable in series. Three components and their assembly are investigated with their differential mode S-parameters. Also, the insertion loss, reflection loss and differential impedance of the components are calculated to analyze their signal transmission characteristics. Each component has approximately $100 \Omega$ of the differential impedance. However, their high-frequency responses such as insertion loss and reflection loss show different characteristics. While the connector and cable present the excellent signal transmission performance, the printed circuit board $(P C B)$ has lower characteristics despite the shortest trace length. The insertion loss and reflection loss of SATA cable assembly are investigated and have a similar tendency with those of the PCB. These results verify that signal transmission properties of the SATA cable assembly are affected significantly by the PCB with the largest loss.
\end{abstract}

Keywords: SATA, Signal transmission, Insertion loss, Reflection loss

\section{Introduction}

Serial advanced technology attachment (SATA) is one of the important serial interface standards in the applications of high-speed interconnection [1]. Particularly, SATA is the most popular ways to transmit and receive the data in PC's storage device at a fast data rate [2].

Recently, the system clock rate has increased continuously to obtain a high-speed operation. Therefore, the electrical problems associated with the signal transmission characteristics occur frequently. It is known that the major causes of the low signal quality in the data transmission system are crosstalk, electromagnetic interference, impedance mismatching and return path discontinuity. But, a single-ended signaling can be difficult to obtain the signal integrity in data transmission system with high wiring density and high-speed operation [3-4]. As a result, a differential signaling has been adopted in high-speed digital applications [5-6].

The differential signaling utilizes two adjacent wires to simultaneously transmit the inphase and out-of-phase. In this technology, it is possible to transfer high-speed data with low voltage operation and low power consumption by detecting the phase difference between the two signals at the receiver. Also, the differential signaling has lower crosstalk, electromagnetic interference, power-ground resonance and common mode noise than the single-ended signaling. In addition, this technology has the advantage when signals cross long cable or between board-to-board interconnections where there will be a voltage difference 
between the transmitter and the receiver local grounds. Therefore, the signal transmission technology in SATA applications has adopted the differential signaling.

In this work, the signal transmission analysis of SATA cable assembly and its components is investigated using a three-dimensional (3D) electromagnetic field simulation based on the finite element method (FEM).

\section{SATA Cable Assembly}

As needs for several gigabit connections are continually increasing, serial bus technologies such as SATA/SAS, HDMI, USB and PCI-Express have emerged to satisfy high bandwidth characteristics. SATA has been widely used as a bus that is traditionally associated with internal storage within a personal computer (PC). As shown in Figure 1, three components in SATA cable assembly are the connector, PCB, and cable, respectively. Also, its cable consists of two data channels and five power/ground lines. The channel 1 is used for SAS and channel 2 corresponds to SATA. Using SATA cable assembly, the main board is interconnected with the data storage unit such as a hard-disk drive, solid-state drive or disc drive.

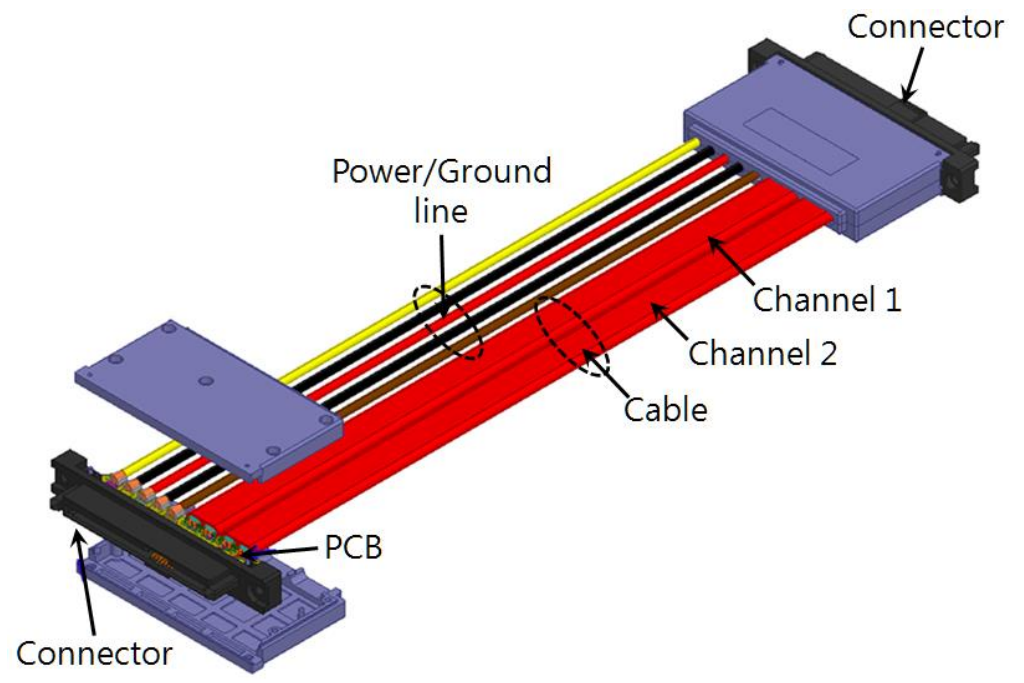

Figure 1. SATA Cable Assembly

The component interconnection of SATA cable assembly is shown in Figure 2. Its cable assembly is connected in series of five components; first connector, first printed circuit board (PCB), cable, second PCB and second connector. The connectors are mounted and soldered onto the pad in the PCB. The cable wires are arranged and soldered through via hole of the PCB. The PCBs provide the short routing traces connecting between the connector and cable.

The material of the connector pin and housing is beryllium copper with a high electrical conductivity and heat-resistant polyimide, respectively. The pitch of the connector pin has a length of $1.27 \mathrm{~mm}$. The pin assignment of the connector is arranged for differential signaling such as ground - signal - signal - ground (GSSG). The connector dimension is designed to satisfy the differential impedance of $100 \Omega$ in this work. 4 layered PCB has a size of $6 \mathrm{~mm} \times 40 \mathrm{~mm}$ and a thickness of $1.3 \mathrm{~mm}$. The differential pairs are used in the routing design of PCB. Their trace width and spacing are determined using two-dimensional electromagnetic calculation (ANSYS 2D 
Extractor). By maintaining a constant spacing between the pairs, its differential impedance can obtain a consistent value $(100 \Omega)$. The two adjacent traces with a width of $0.14 \mathrm{~mm}$ and a separation of $0.18 \mathrm{~mm}$ are routed in this work. Also, designing the trace length as short as possible, its signal loss on PCB can be reduced and then its signal transmission will be better. In the cable, the differential signaling consists of two adjacent centered coppers cladding with a circular dielectric of polyolefin and its length is $160 \mathrm{~mm}$.

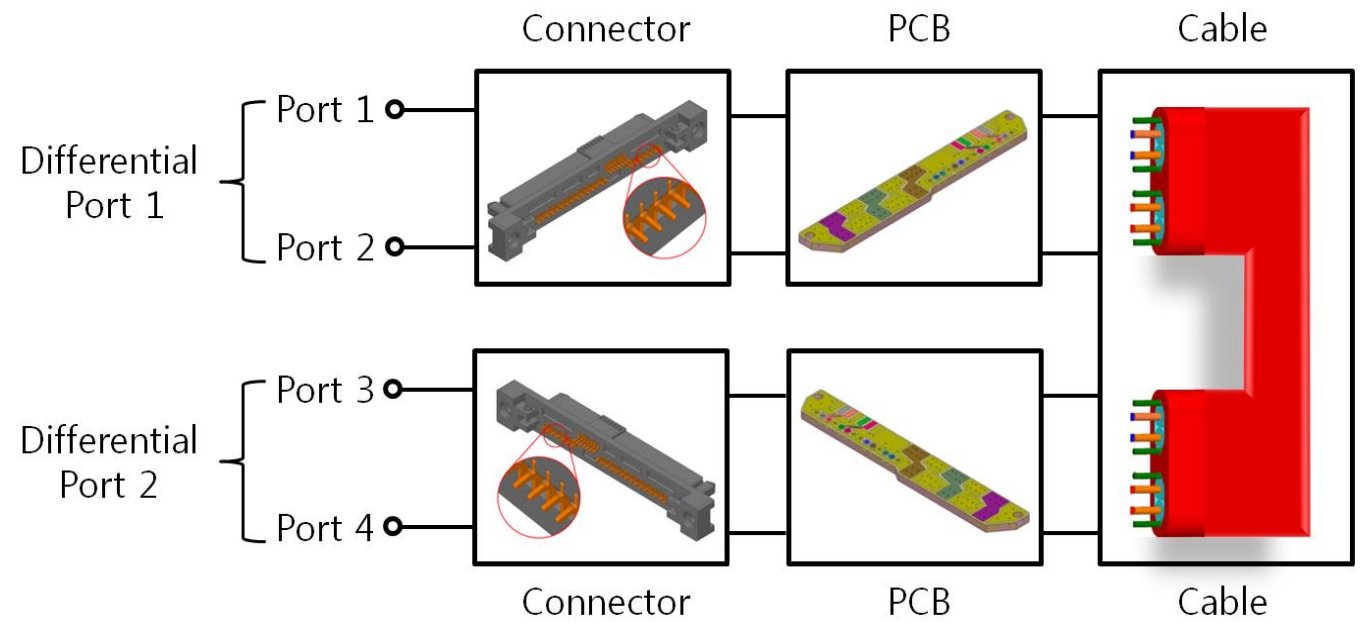

Figure 2. Component Interconnection of SATA Cable Assembly

\section{Signal Transmission Analysis}

As shown in Figure 2, 4-port S-parameters of SATA cable assembly and the components are calculated to investigate their signal transmission characteristics. In SATA applications, the concept of the S-parameter in a single-ended signaling is extended to differential interconnections. Therefore, the signal propagation and reflection are described using differential mode S-parameter. The single-ended 4-port S-parameter is defined by (1)

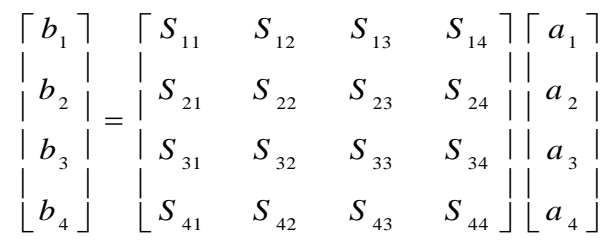

where $a_{i}$ and $b_{i}$ are normalized incident and reflected waves. The differential mode $S$ parameters are extracted from mode conversion equation [7] and are expressed by (2)

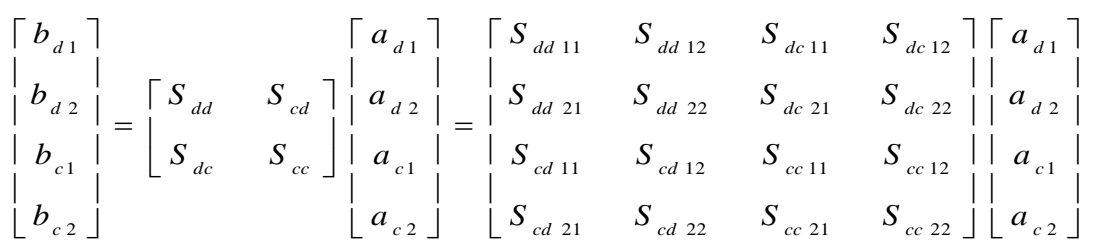


where the four $\mathrm{S}$ blocks in the left matrix present a two-by-two submatrix. $\mathrm{S}_{\mathrm{dd}}$ corresponds to the differential mode $S$-parameters while $S_{\mathrm{cc}}$ presents the common mode parameters. The other two quadrants correspond to the mode conversion terms. The transformation equation between the single-ended S-parameters and differential mode S-parameters can be express by (3) $[8]$

$$
\begin{aligned}
& S_{d d 11}=\frac{1}{2}\left(S_{11}-S_{21}-S_{12}+S_{22}\right) \\
& S_{d d 12}=\frac{1}{2}\left(S_{13}-S_{23}-S_{14}+S_{24}\right) \\
& S_{d d 21}=\frac{1}{2}\left(S_{31}-S_{41}-S_{32}+S_{42}\right) \\
& S_{d d 22}=\frac{1}{2}\left(S_{33}-S_{43}-S_{34}+S_{44}\right)
\end{aligned}
$$

where $S_{\mathrm{dd} 21}$ is the ratio of the differential output from port 2 to the differential input into port 1. In this work, the differential reflection loss $\left(S_{\mathrm{dd} 11}\right)$ and differential insertion loss $\left(\mathrm{S}_{\mathrm{dd} 21}\right)$ are investigated to analyze the signal transmission characteristics of the components and their assembly using the electromagnetic simulation (ANSYS HFSS).

In general, the signal transmission may be degraded by impedance discontinuities [9]. In this work, the differential impedance $\left(Z_{\text {diff }}\right)$ is investigated to analyze the impedance mismatching of the component and is calculated using (4) and (5) [10]

$$
\begin{aligned}
& Z_{\text {odd }}=\sqrt{\frac{L_{s}-L_{m}}{C_{s}+C_{m}}} \\
& Z_{\text {diff }}=2 \times Z_{\text {odd }}
\end{aligned}
$$

where $Z_{\text {odd }}$ is the odd mode impedance, $L_{s}$ is the self inductance, $L_{m}$ is the mutual inductance, $\mathrm{C}_{\mathrm{s}}$ is the self capacitance and $\mathrm{C}_{\mathrm{m}}$ is the mutual capacitance. The differential impedances of the components and their assembly are calculated using ANSYS Q3D. In the simulation, GSSG configuration is assigned for differential pairs. In the simulation of the connector, the electrical conductivity of beryllium copper and the dielectric constant of polyimide are assigned to be $4.7 \times 10^{7} \mathrm{~S} / \mathrm{m}$ and 3.7 , respectively. In the simulation of the cable and PCB, the electrical conductivity of copper is $5.8 \times 10^{7} \mathrm{~S} / \mathrm{m}$ and the dielectric constant of insulating materials is set to be 2.3 and 4.4 . 


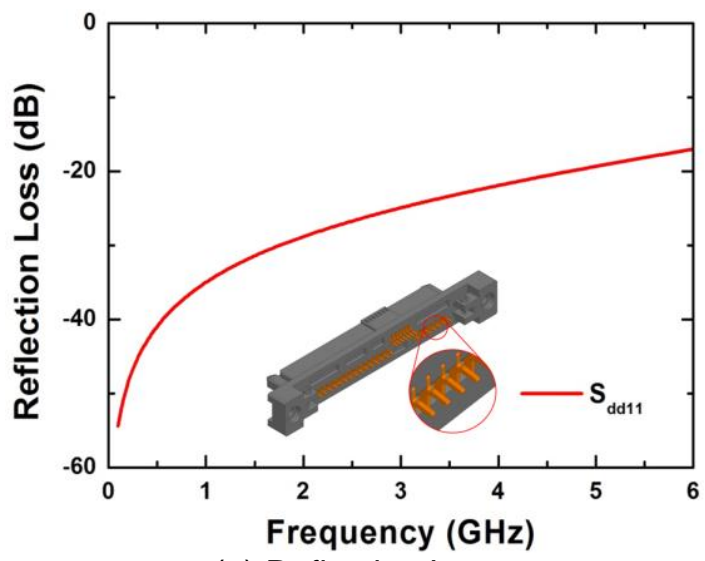

(a) Reflection Loss

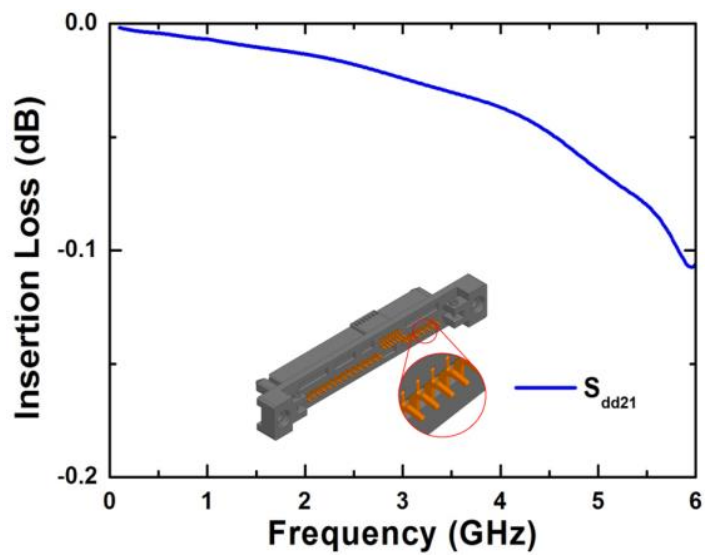

(b) Insertion Loss

Figure 3. Signal Transmission Characteristics of Connector

Table 1. Differential Impedance of Connector

\begin{tabular}{|c|c|}
\hline Parameter & Value \\
\hline $\mathrm{L}_{\mathrm{s}}(\mathrm{nH})$ & 3.07 \\
\hline $\mathrm{L}_{\mathrm{m}}(\mathrm{nH})$ & 1.20 \\
\hline $\mathrm{C}_{\mathrm{s}}(\mathrm{pF})$ & 0.47 \\
\hline $\mathrm{C}_{\mathrm{m}}(\mathrm{pF})$ & 0.18 \\
\hline $\mathrm{Z}_{\mathrm{diff}}(\Omega)$ & 107.3 \\
\hline
\end{tabular}

The signal transmission characteristics of the reflection loss $\left(\mathrm{S}_{\mathrm{dd} 11}\right)$ and insertion loss $\left(S_{\mathrm{dd} 21}\right)$ in the connector are calculated as shown in Figure 3. Low reflection loss of less than $20 \mathrm{~dB}$ is obtained in most frequency ranges as shown in Figure 3 (a). Also, the connector has the excellent insertion loss with approximately $-0.1 \mathrm{~dB}$ at the maximum frequency. These results verify that the connector has a superior performance in its signal transmission characteristics. Table 1 shows the differential impedance of the connector. As predicted from the results of Figure 3, the connector is well matched to the reference impedance of $100 \Omega$.

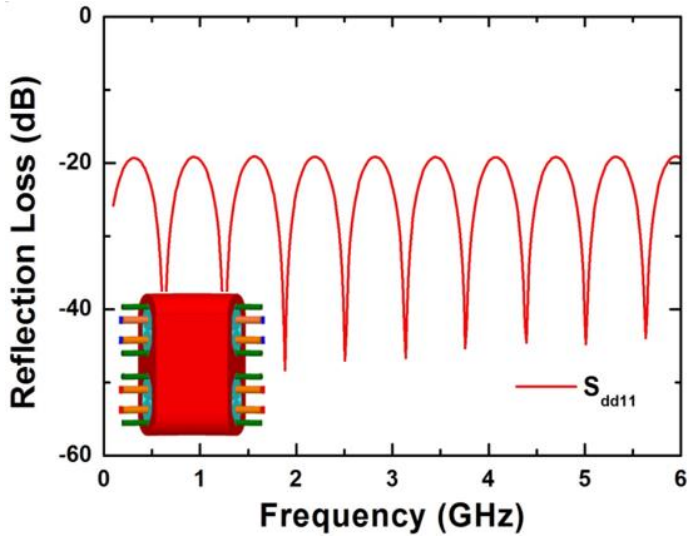

(a) Reflection Loss

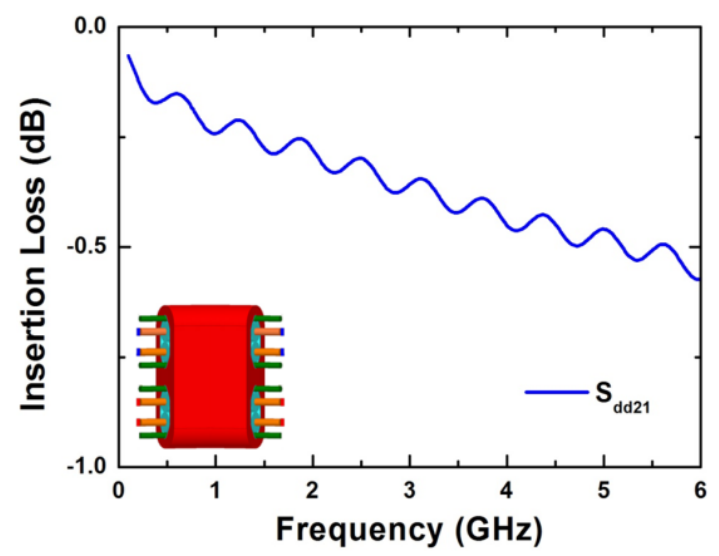

(b) Insertion Loss

Figure 4. Signal Transmission Characteristics of Cable 
Table 2. Differential Impedance of Cable

\begin{tabular}{|c|c|}
\hline Parameter & Value \\
\hline $\mathrm{L}_{\mathrm{s}}(\mathrm{nH})$ & 0.84 \\
\hline $\mathrm{L}_{\mathrm{m}}(\mathrm{nH})$ & 0.34 \\
\hline $\mathrm{C}_{\mathrm{s}}(\mathrm{pF})$ & 0.15 \\
\hline $\mathrm{C}_{\mathrm{m}}(\mathrm{pF})$ & 0.04 \\
\hline $\mathrm{Z}_{\mathrm{diff}}(\Omega)$ & 102.6 \\
\hline
\end{tabular}

Figure 4 shows the differential reflection loss and insertion loss of the cable. Similar to the signal transmission results of the connector, the cable has both low losses. The reflection loss of the connector increases continuously with the frequency, while that of the cable shows the periodic resonance characteristics to the frequency. The different results of both components are associated with their structure such as a length and a size. The differential impedance of the cable is calculated and presented in Table 2. Compared to the connector, the cable has a value closer to the impedance matching.

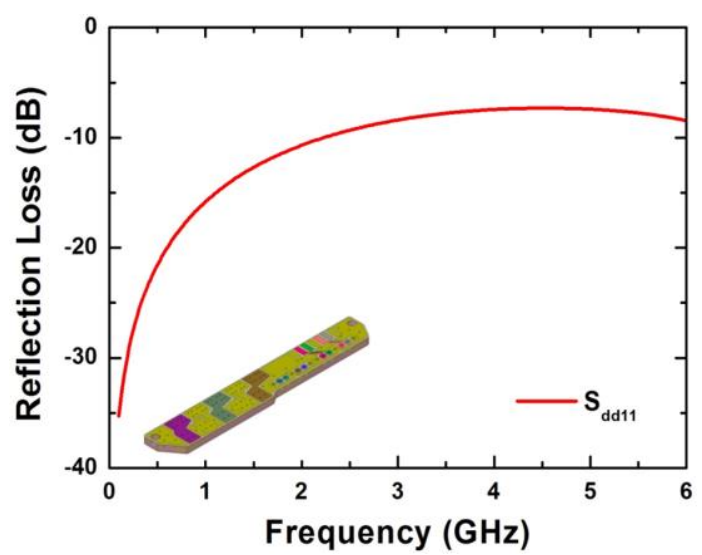

(a) Reflection Loss

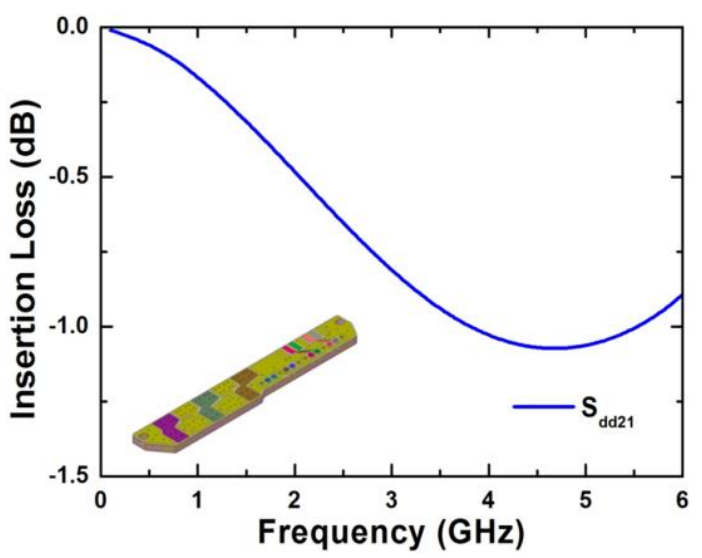

(b) Insertion Loss

Figure 5. Signal Transmission Characteristics of PCB

Table 3. Differential Impedance of PCB

\begin{tabular}{|c|c|}
\hline Parameter & Value \\
\hline $\mathrm{L}_{\mathrm{s}}(\mathrm{nH})$ & 350.3 \\
\hline $\mathrm{L}_{\mathrm{m}}(\mathrm{nH})$ & 59.8 \\
\hline $\mathrm{C}_{\mathrm{s}}(\mathrm{pF})$ & 99.8 \\
\hline $\mathrm{C}_{\mathrm{m}}(\mathrm{pF})$ & 8.1 \\
\hline $\mathrm{Z}_{\mathrm{diff}}(\Omega)$ & 103.8 \\
\hline
\end{tabular}

The differential reflection loss and insertion loss of the PCB are shown in Figure 5. Compared to the connector and cable, the PCB has the shortest trace length of about $2.2 \mathrm{~mm}$ from the pad to via. Therefore, it is expected that the PCB has low loss in signal transmission characteristics. But, a relatively large reflection loss of more than $-10 \mathrm{~dB}$ is shown in Figure 5 (a). Also, an insertion loss of $-1 \mathrm{~dB}$ is reached at high frequency ranges in Figure 5 (b). Table 3 shows the differential impedance of the PCB. For high-speed operation, the differential impedance of the signal traces on PCB is designed to be $100 \Omega$. Its differential impedance of 
103.8 $\Omega$ is achieved in this work. However, it is difficult to demonstrate the differential impedance of $100 \Omega$ even at the pad and via because they have relatively large dimensions by a limitation of a fabrication process. Therefore, there are the impedance discontinuities around the pad and via and then the signal fidelity on the PCB is deteriorated.

As shown in Figure 3 (b), the connector shows a very low insertion loss of less than $0.1 \mathrm{~dB}$ in almost frequency range. Also, the cable has a relatively low loss in Figure 4 (b) although it has long length of $160 \mathrm{~mm}$. But, the PCB has largest insertion loss in Figure 5 (b) despite the shortest trace length. Therefore, it is expected that the insertion loss of SATA cable assembly will increase due to relatively large loss of the PCB.

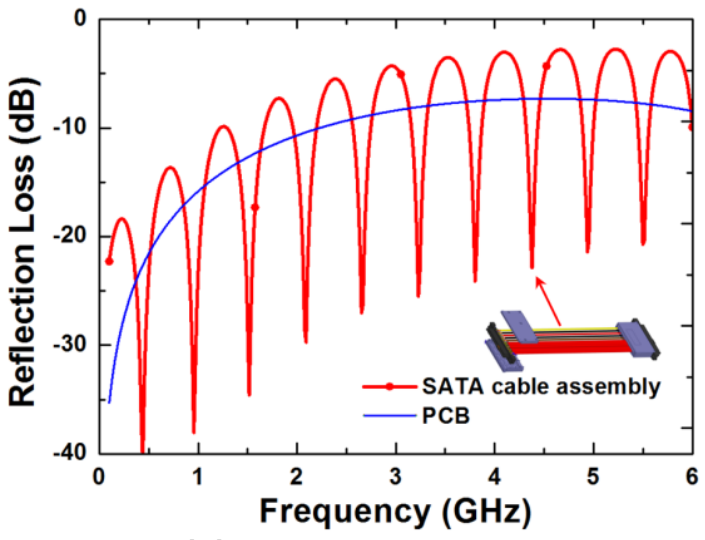

(a) Reflection Loss

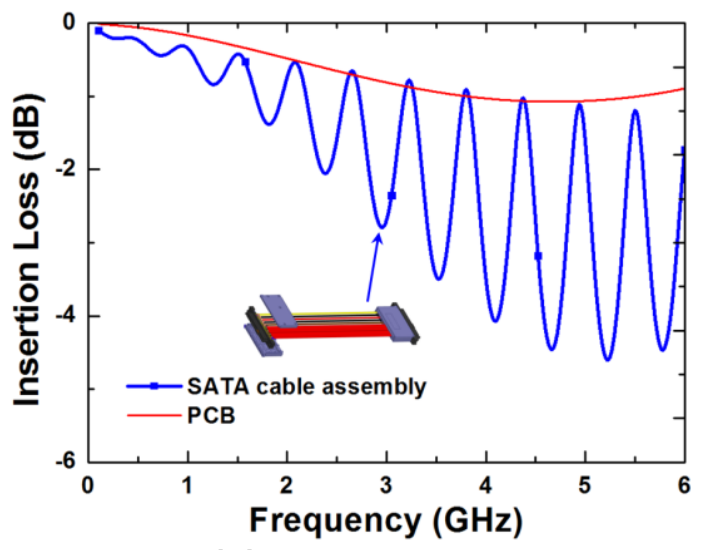

(b) Insertion Loss

Figure 6. Signal Transmission Characteristics of SATA Cable Assembly

The signal transmission calculation of SATA cable assembly is performed using the circuit simulator (ANSYS Designer). The circuit simulator includes all 4-port S-parameter models of connectors, PCBs and cable as shown in Figure 2. Figure 6 shows the signal transmission characteristics of SATA cable assembly. The reflection loss of SATA cable assembly has almost same tendency with that of the PCB in Figure 6 (a). Also, the similar effects occur in the insertion loss as shown in Figure 6 (b). These results demonstrate that the signal transmission characteristics of SATA cable assembly are mainly determined by the worst performance of their components. In this work, the PCB degrades substantially the signal quality of the whole system.

\section{Conclusion}

The signal transmission characteristics of SATA cable assembly are investigated using electromagnetic field simulation. The differential reflection loss and insertion loss of the components and their assembly are calculated. The connector and cable show the excellent signal transmission characteristics, while the PCB presents relatively large loss despite the short signal length. The differential impedance is investigated to analyze the impedance mismatching of the component. The differential impedance of all components is well matched to the reference value. However, there are the impedance discontinuities around the pad and via on PCB. Because of these effects, PCB's signal transmission characteristics are degraded. Therefore, the low performance of PCB significantly influences the signal integrity of SATA cable assembly in this work. 


\section{Acknowledgements}

This research was supported by Basic Science Research Program through the National Research Foundation of Korea (NRF) funded by the Ministry of Education (2012R1A1A2008065) and also supported by the International Science and Business Belt Program through the Ministry of Science, ICT and Future Planning (former Education, Science and Technology) (2012K001564).

\section{References}

[1] "Serial ATA International Organization", Serial ATA Revision 3.0, Serial ATA International Organization, (2009).

[2] J. Vana, A. Barr, R. Scherer and A. Joshi, "High Speed I/O Test Cable Assembly Interfaces for Next Generation Multi-Gigabit Serial Protocols", Proceedings of the Internal Test Conference, (2009), pp. 1.

[3] J. Chandrasekhar, E. Engin, M. Swaminathan, K. Uriu and T. Yamada, "Noise Induced Jitter in Differential Signaling", Proceedings of IEEE Electronic Components and Technology Conference, (2008), pp. 17551761.

[4] N. Na, J. Audet and D. Zwitter, "Discontinuity Impacts and Design Considerations of High speed Differential Signals in FC-PBFA Packages with High Wiring Density", Proceedings of IEEE Electrical Performance of Electronic Packaging, (2005), pp. 107-110.

[5] W. L. Yuan, H. P. Kuah, C. K. Wang, A. Y. S. Sun, W. H. Zhu, H. B. Tan and A. D. Muhamad, "HighSpeed Differential Interconnection Design for Flip-Chip BGA Packages", Proceedings of IEEE Electronics Packaging Technology Conference, (2006), pp. 76-81.

[6] M. Cocchini, J. Fan, B. Archambeault, J. L. Knighten, X. Chang, J. L. Drewniak, Y. Zhang and S. Connor, "Noise Coupling Between Power/Ground Nets Due To Differential Vias Transitions in a Multilayer PCB", Proceedings of IEEE International Symposium on Electromagnetic Compatibility, (2008), pp. 1-6.

[7] D. G. Kam, H. Lee and J. Kim, "Twisted Differential Line Structure on High-speed Printed Circuit Boards to Reduce Crosstalk and Radiated Emission”, IEEE Transactions on Advanced Packaging, vol. 27, no. 4, (2004), pp. 590-596.

[8] Anritsu, "Three and Four Port S-parameter Measurements", Application Note, (2008).

[9] W. L. Yuan, H. P. Kuah, C. K. Wang, A. Y. S. Sun, W. H. Zhu, H. B. Tan and A. D. Muhamad, "HighSpeed Differential Interconnection Design for Flip-Chip BGA Packages”, Proceedings of IEEE Electronics Packaging Technology Conference, (2006), pp. 76-81.

[10] S. C. Thierauf, "High-speed Circuit Board Signal Integrity", Artech House, Boston, (2004), pp. 149-170.

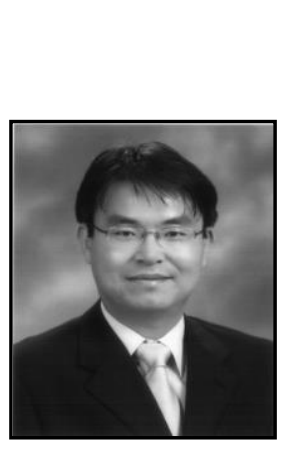

\section{Author}

Moonjung Kim, he received the B.S. degrees in electronic engineering from Kyungpook National University, Daegu, Republic of Korea, in 1997 and the M.S. and Ph.D. degree in electrical and electronics engineering from Korea Advanced Institute of Science and Technology, Daejeon, in 2003. Since September 2006, he has been with the Division of Electrical Electronics and Control Engineering, Kongju National University, Chungnam, Korea, as a faculty member. His current research interests include the signal/power integrity and signal transmission analysis in the highspeed package/board/system. 